\title{
Experimental Analysis of Mechanical and Electrical Characteristics of Metal-Coated Conductive Spheres for Anisotropic Conductive Adhesives (ACAs) Interconnection
}

\author{
Woon-Seong Kwon and Kyung-Wook Paik, Member, IEEE
}

\begin{abstract}
An accurate characterization for the deformation behavior of conductive particles is important: 1) to understand the anisotropic conductive adhesive (ACA) interconnection and 2) to optimize the ACA bonding parameter. This paper introduces an experimental technique, which has been developed to allow continuous monitoring of deformation characteristics of a single conductive particle. The load-deformation curve of a single conductive particle is measured, which provides the quantitative estimation of the mechanical and electrical characteristics of metal-coated polymer spheres used in ACAs. Based on the load-deformation result of a single conductive particle and the number of trapped particles on a bump, equivalent spring models are used to predict the deformation degree of conductive particles after flip chip assembly. For two kinds of conductive particles with different polymer cores, the mechanical and electrical characteristics of ACA interconnection were studied. Such results are used to further achieve a more sophisticated approach of the ACA bonding process and contact reliability.
\end{abstract}

Index Terms-Anisotropic conductive adhesive (ACA), conductive particles, contact resistance, flip chip bonding, load-deformation characteristics.

\section{INTRODUCTION}

$\mathbf{I}$ N RECENT years, anisotropic conductive adhesives (ACAs) have emerged as an important joining technology, such as advanced display assembly, contactless smart-card module assembly, and bare chip attach on flexible and rigid substrates. Advanced communications function (ACF) and its unique properties have been accepted because of the requirements of liquid crystal displays (LCDs) and flexible package applications. In detail, LCD devices and flexible substrate cannot be exposed to high temperatures because of the thermal instability of the liquid crystal and polarizers $\left(T<120^{\circ} \mathrm{C}\right.$ is required $)$ and the dimensional instability of a flexible printed circuit (FPC), respectively. Moreover, the connection pitch of the electrodes of the driver integrated circuit (IC) has been decreased from $160 \mu \mathrm{m}$ to $35 \mu \mathrm{m}$, and the number of output terminal electrodes per IC

Manuscript received August 26, 2004; revised May 12, 2005. This work was recommended for publication by Associate Editor K. Jonnalagadda upon evaluation of the reviewers' comments.

The authors are with the Department of Materials Science and Engineering, Korea Advanced Institute of Science and Technology, Daejon 305-701, Korea (e-mail: wskwon@kaist.ac.kr).

Color versions of Figs. 1, 3, 5-8, and 10-12 are available online at http://ieeexplore.ieee.org.

Digital Object Identifier 10.1109/TCAPT.2006.880513

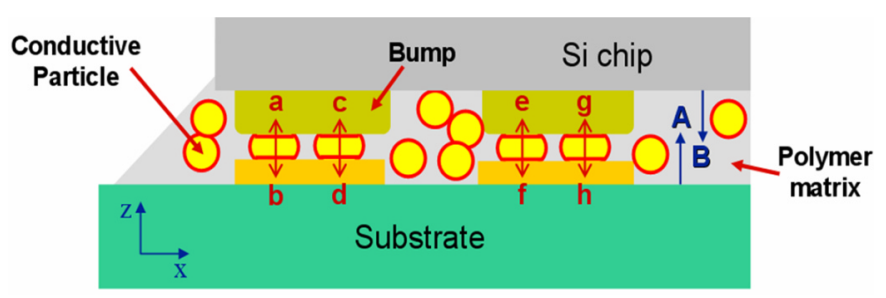

(a)

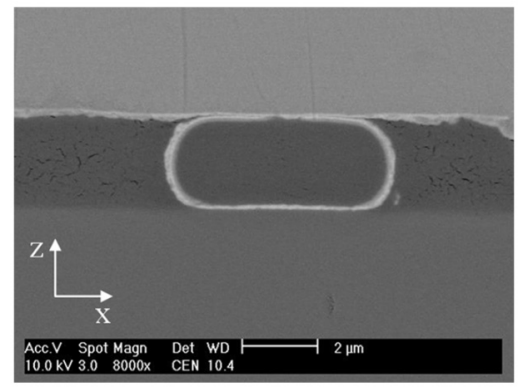

(b)

Fig. 1. (a) Schematic diagram of the conduction mechanism through anisotropic conductive films (ACF's). A, B: Contractive forces of $\mathrm{ACF}_{\mathrm{s}}$, a-h: Repulsive forces of compressed conductive particles, (b) Cross-sectional view of compressed conductive particles between a hard nickel bump and a substrate pad.

has been increased from 200 points per IC to 500 points per IC, making it possible to realize high-resolution display modules for notebook PCs and mobile products. Anisotropic electrically conductive materials have been applied in order to cope with the demands of such low temperature assembly, high input/output (I/O) number and fine interconnection pitch for LCD and flex substrate applications.

ACAs consist of an adhesive polymer matrix and fine conductive fillers of metallic, or metal-coated polymer, particles, and when used for IC assembly provide electrical conduction as well as mechanical interconnections [1]. As schematically shown in Fig. 1, electrical conduction is established by the deformation of the conductive particles trapped between chip bumps and substrate pads in the $z$-axis of the adhesives perpendicular to the plane of the board, while electrical isolation is maintained in the plane of the adhesive layer [2]-[4]. Therefore, ACAs main function as mechanical bonding based on its adhesion to both chip and substrate and electrical conduction through the deformation of conductive particles between chip bumps and substrate 
pads. Furthermore, ACA contacts are mechanically constructed by both the solidification of the adhesive polymer matrix caused by curing and the thermo-mechanical stress during a cooling process, which builds up the sufficient contractive force to establish stable and low resistance electrical connection [5], [6].

In general, it has been found that the conductive particles played an important role in conduction establishment in ACA joints, while the deformation degree of the conductive particles is the key factor. The required deformation degree of the particle is controlled by bonding conditions and tool accuracy.

The particle deformation can increase the contact area of the particles, thus the electrical conductivity. Accordingly, a fundamental understanding about the deformation characteristics of single and multi-particle systems is very important to understand ACA's contact and to obtain a high bonding quality for ACA's assembly. In particular, mechanical properties of polymer core of a conductive particle may also affect the deformation degree and electrical characteristics in ACA joint. However, there was not a feasible way to measure the deformation characteristics of single conductive particle due to its very small size $(3-10 \mu \mathrm{m})$. Therefore, very few experimental investigations about the deformation of single conductive particle have been reported.

In this study, load-deformation behavior of conductive particles with different polymer core was quantitatively characterized by using a nano-mechanical testing system. Based on the deformation behaviors of each conductive particle, mechanical and electrical properties of ACA joint were studied to improve the bonding quality, process throughput, and reliability.

\section{Mechanical Contact Models OF ACA INTERCONNECTIONS}

Some models of ACA electrical interconnection have been proposed. These models fundamentally deal with contact area, degree of deformation, bonding force, and contact resistance. And the magnitude of particle deformation is determined by applied force, contact hardness, diameter, and the number of conductive particles. In addition, models were mathematically formulated based on the Hertzian contact mechanics theory (linear elastic) or numerically analyzed with finite-element method (FEM) (plastic).

Actually, an accurate prediction of a contact resistance model should deliberately consider various characteristics such as particle deformation, contact materials (bump and substrate terminal), contact interface, and coating layer rupture.

\section{A. Elastic Contact Under a Small Deformation}

Fig. 2 represents a schematic deformation of a half conductive particle between a bump and a pad. The dashed and solid lines show the situation before and after the deformation, respectively. Considering a full conductive particle, total deformation $\Delta$ is $2 \cdot \delta$. For an elastic contact under a small deformation, the relation between contact area $A$ and applied force $F$ can be generally determined by using the Hertz's theory of elastic contact [7].

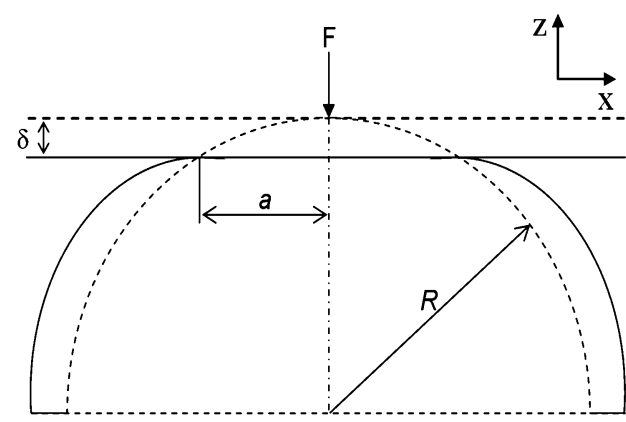

Fig. 2. Schematic deformation of a half conductive particle when a contact load is applied.

With the assumption of perfectly elastic deformation $\delta$ at small contact load and Hertz's contact theory [7], $\delta$ can be expressed as

$$
\delta=\left(\frac{9}{16} \frac{F^{2}}{R E^{* 2}}\right)^{1 / 3}
$$

where the effective elastic modulus, $E^{*}$, is

$$
\frac{1}{E^{*}}=\frac{1-\nu_{p}^{2}}{E_{p}}+\frac{1-\nu_{b}^{2}}{E_{b}}
$$

and $F$ is the force applied to single conductive particle, $R$ is the radius of conductive particle, $E$ is the elastic modulus, $v$ is the poisson's ratio, $p$ and $b$ represent the particle and bump, respectively.

The elastic contact radius $a$ is given by

$$
a^{2}=R \delta \text {. }
$$

Therefore

$$
a=\left(\frac{3 F R}{4 E^{*}}\right)^{1 / 3}
$$

where the contact surface is a circular shape. Therefore, the contact area is obtained as

$$
A=\pi a^{2}=\pi\left(\frac{3 F R}{4 E^{*}}\right)^{2 / 3} .
$$

\section{B. Elastic-Plastic Contact Under a Large Deformation}

As described above, a small contact load causes the conductive particle to deform elastically according to the Hertz's theory. With further increase of contact loads, the conductive particle starts deforming plastically. Under this condition, the elastic contact model may underestimate the contact area. And if a substantial portion of deformation is plastic, a significant error can be introduced into the load-deformation and contact area-deformation relationship.

As the contact load increases, plastic deformation builds up with some elastic deformation still remaining. This is an elasticplastic deformation. Since the total deformation is composed of a mixture of the elastic and plastic components at this stage, deformation characteristics such as contact load, contact area, and degree of deformation become complex. With the further increase of loads, plastic deformation dominates.

The contact area of a conductive particle depends not only on the applied force $F$ and deformation $\Delta$ but also on the degree 
of plastic deformation. Accordingly, the dependence of contact area on the plastic deformation is

$$
A=\pi a^{2}=\pi(g R \delta), \quad(1 \leq g \leq 2)
$$

$g=1:$ linear elastic deformation;

$1\langle g\langle 2$ : elastic-plastic deformation;

$g=2$ : fully plastic deformation.

\section{EXPERIMENTAL}

\section{A. Experimental Apparatus}

In this study, deformation measurements of single conductive particles described were performed using a commercially available apparatus, Nano Indenter XP with a flat punch (Nano Instruments, Oakridge, USA). In general, the nano-indenter uses the Berkovich tip with three-sided diamond pyramid. However, in this experiment, the diamond flat punch shown in Fig. 3 was specially used to measure the deformation characteristics of a single conductive particle. A single conductive particle becomes compressed between a flat punch and a glass substrate. Quantitative measurement of mechanical deformation of a single conductive particle can be obtained using this equipment which is capable of operating at a micronewton $(\mu \mathrm{N})$ range of loads and subnanometer range of displacement.

The flat punch in this experiment requires precise calibration, especially flat punch planarity. The planarity calibration was evaluated by the indentation impressions appeared after the indentation of four-sided flat punch onto aluminum single crystal. The indentation condition, where all the four sides of $100 \times 100 \mu \mathrm{m}^{2}$ rectangular flat punch surface are well pressed, is chosen as a good flat punch condition with superior planarity. Defects such as dust or external impurities should be removed on the flat punch surface, because these may affect the evaluation of deformation characteristics of the tested conductive particle.

In order to maintain a better control upon the machine operations during the measurements, no thermal drift correction has been implemented in the set of experiments described in this paper. Measurements were conducted in sequential steps: 1) approach of flat punch to the upper surface of a conductive particle, 2) loading with a rate of $15 \mathrm{~nm} / \mathrm{s}$, and 3) $100 \%$ unloading with a rate of $15 \mathrm{~nm} / \mathrm{s}$.

\section{B. Conductive Particles and ACA Materials}

Two kinds of conductive particle with different polymer core were prepared to investigate the effect of polymer core on the particle deformation and contact resistance of ACA interconnection. Adhesive resins prepared by formulating epoxy resin, curing agents, and some additives were mixed with conductive particles and transferred to a dry film format using film coater. ACAs used in this study are about $40 \mu \mathrm{m}$ in thickness.

\section{Testchips and Bonding Process}

The dimension of the silicon chips was $10 \mathrm{~mm} \times 10 \mathrm{~mm} \times$ $0.6 \mathrm{~mm}$. The electroless Ni bumps were formed on the Al pad of the chip. The number of bumps was 120 and the bump pitch was $250 \mu \mathrm{m}$. Fig. 4 shows the SEM pictures of electroless nickel

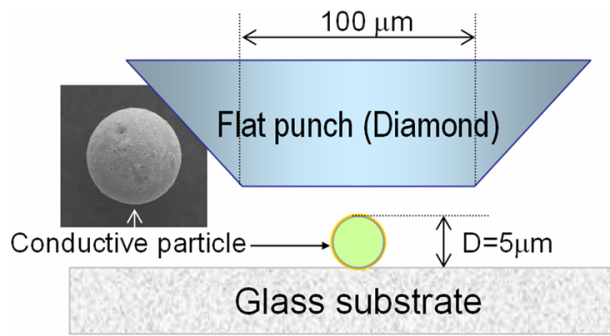

Fig. 3. Schematic diagram of experimental apparatus for the load-deformation measurement of a small size particle $(D=5 \mu \mathrm{m})$.

bumps on the testchip. Effective contact area for the deformation of conductive particles is practically top surface of electroless Ni bumps.

The conductive particles studied are nickel $(0.05 \mu \mathrm{m}) / \mathrm{gold}$ $(0.05 \mu \mathrm{m})$ coated polymer spheres with two different polymer cores. The average conductive particle size was $5.0 \mu \mathrm{m}$. The $0.7-\mathrm{mm}$-thick glass substrate had a thin gold pattern. The reason for selecting the gold patterned glass as a substrate was to eliminate the uneven particle deformation caused by the metal trace on the circuit boards.

After ACFs were then laminated on the glass substrate, the chip and the substrate were aligned and finally bonded under various bonding pressures at a temperature of $180^{\circ} \mathrm{C}$ for $15 \mathrm{~s}$.

The contact resistance of a single ACA joint was measured by using the four point probe method and the schematic circuitry is shown in Fig. 5. In this measurement, constant dc current was applied to the circuit and the voltage was read from the KEITHLEY 236 source measure unit. Then, the contact resistance was obtained simply by using Ohm's law, $R=V / I$.

\section{RESUlTS AND DISCUSSION}

\section{A. Load Versus Deformation Analysis of Single Conductive Particle}

Compressive load versus deformation curves of a single conductive particle were shown in Fig. 6. Each curve represents the deformation behavior of each conductive particle with a different polymer core (type A and B). The nonlinearity is mainly due to the change of the contact area during the particle compression and to the viscoelastic properties of the polymeric core material. During normal compression, single conductive particle undergoes both elastic and plastic deformation. These loading curves describe the compression hardness of the core material. Accordingly, the discrepancy between these two curves indicates the softer nature of conductive particle with B type core material when compared with the A type core particle. In other words, a conductive particle with the softer core requires lower force to induce a comparable particle deformation. As shown in Fig. 6, contact forces of $30 \mathrm{mN}$ and $10 \mathrm{mN}$ were, respectively, required to compress the conductive particles with A and B type core material into $3 \mu \mathrm{m}$. It is clearly shown that the polymeric core material of a conductive particle significantly affects the deformation degree of a conductive particle. This load-deformation curve provides the quantitative bonding parameter guideline for ACA interconnection. 


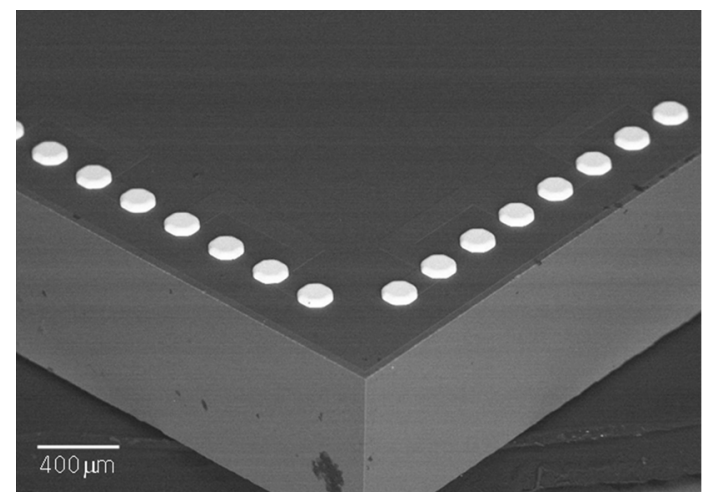

(a)

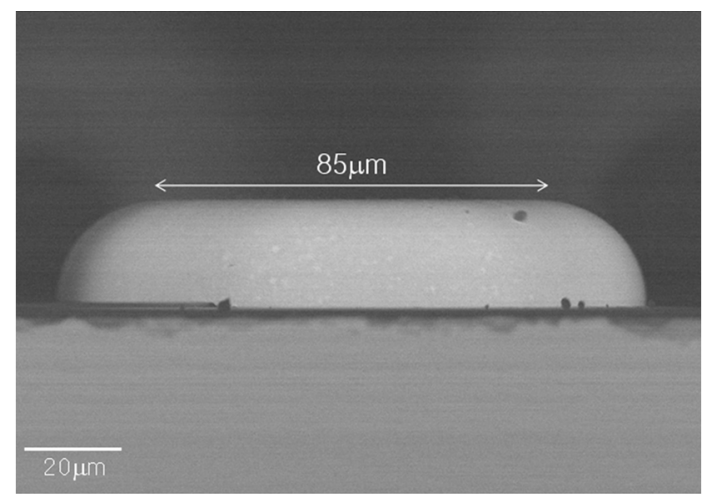

(b)

Fig. 4. SEM pictures of (a) electroless Ni bumped flip chip and (b) electroless Ni/Au bumps on a chip.

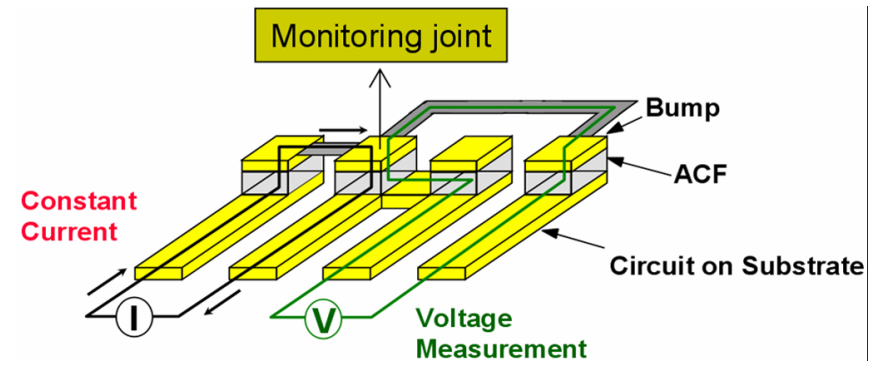

Fig. 5. Four point probing method for contact resistance measurement of single joint of ACA interconnection.

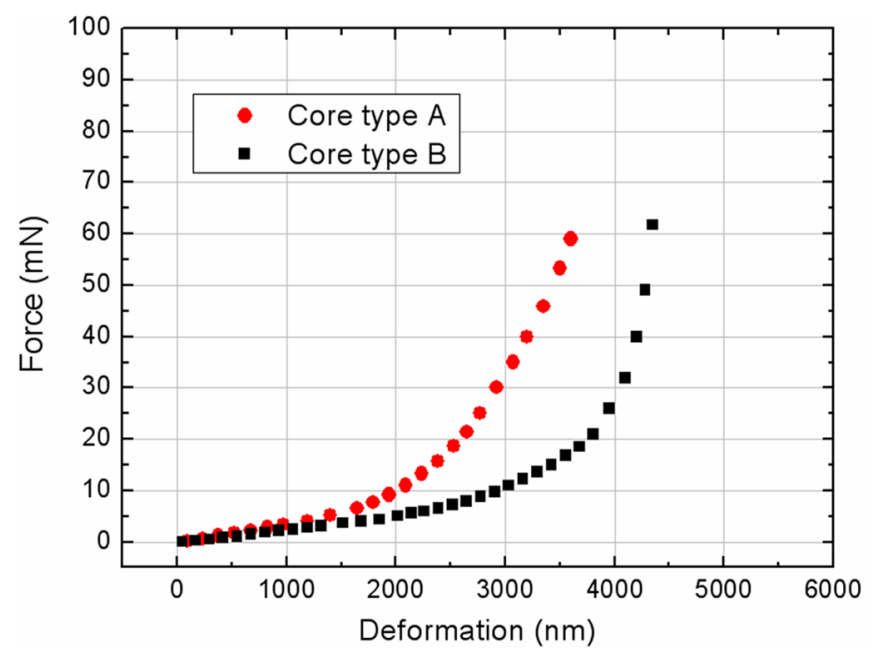

Fig. 6. Load-deformation curves of a single conductive particle ( $D=5 \mu \mathrm{m})$ with two types of polymer core.

\section{B. Prediction and Validation of Bonding Parameter}

The volume, size, and core material of conductive particles in ACA vary with the application area, bonding requirement, and manufacturer. Moreover, the bonding force, which is the very critical parameter for particle deformation, is determined by the particle trapping density on a bump and core hardness of conductive particles in ACA.

Fig. 7 presents the equivalent spring model for a conductive particle to estimate the contact force of ACA interconnection. Based on the measured deformation versus load results of a single conductive particle, the total bonding load of thermode
Single spring model Assembly level equivalent spring model
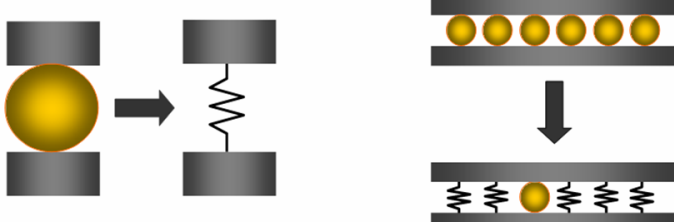

$$
F_{\text {total }}=N_{p}(\text { particles }) \times F_{p}(\text { load } / \text { particle })
$$

Fig. 7. Schematic spring model for the bonding force estimation based on deformation characteristics of the single particle.

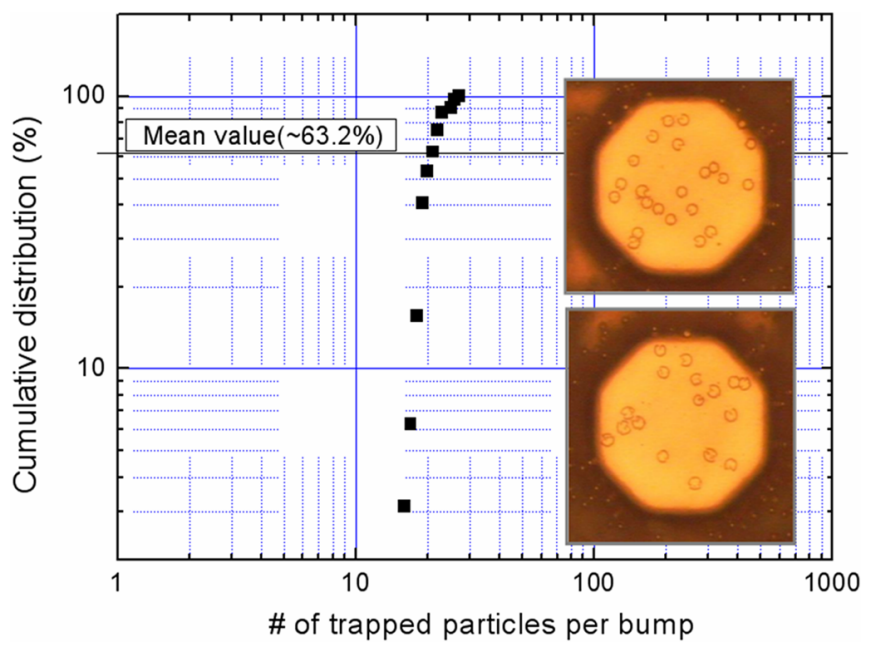

Fig. 8. Cumulative distribution of the number of trapped particles on a bump and optical microscopy of trapped conductive particles.

for a specific particle deformation can be estimated using the spring model in Fig. 7.

By examining the trapped particles in the contact area with an optical microscope through the thin glass substrate, we can easily count the number of particles on a bump. Fig. 8 shows the cumulative distribution of the number of trapped particles between a bump and a substrate terminal. As shown in Fig. 8, the randomly distributed particles in ACA lead to the variation in the number of trapped particles. Since the number of particles in the contacts was statistically distributed, the number of 


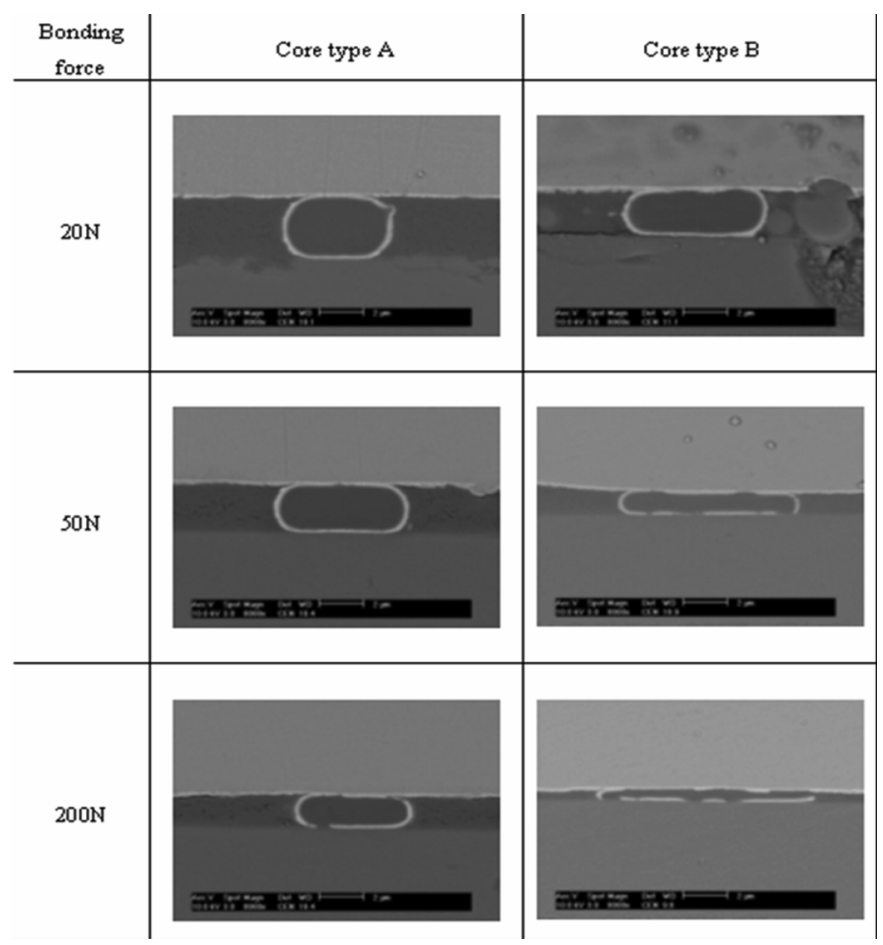

Fig. 9. Deformed conductive particle (particle diameter: $5 \mu \mathrm{m}$ ) between a bump and a substrate under bonding load of (a) $20 \mathrm{~N}$, (b) $50 \mathrm{~N}$, and (c) $200 \mathrm{~N}$.

TABLE I

Contact Results of the PARTiCles WITH the ChIP AND Substrate

${ }^{1}$ Divided by the average number of paticles, ${ }^{2}$ Estimated from Fig. $6,{ }^{3}$ Measured by SEM and OM

\begin{tabular}{l|ccc|ccc}
\hline & \multicolumn{3}{|c|}{ Core type A } & \multicolumn{3}{c}{ Core type B } \\
\hline Total bonding pressure (N/chip) & 25 & 50 & 200 & 25 & 50 & 200 \\
\hline Load per particle $^{1}(\mathrm{mN} /$ particle) & 10.4 & 20.8 & 83.3 & 10.4 & 20.8 & 83.3 \\
\hline Estimated deformation $^{2}(\mu \mathrm{m})$ & 2.0 & 2.6 & 3.2 & 2.7 & 3.8 & 4.5 \\
\hline Measured deformation $^{3}(\mu \mathrm{m})$ & 1.7 & 2.5 & 3.1 & 2.8 & 3.7 & 4.4 \\
\hline
\end{tabular}

particles trapped per bump was adopted as a mean value. The chip had 120 bumps and a measured average particle density of 20 per bump. This gives a total number $\left(N_{p}\right)$ of particles of 2400, which means the number of conductive particles to be compressed during bonding process. The load $\left(F_{p}\right)$ required to compress a single conductive particle was previously described at the Fig. 7. Therefore, the total load to compress conductive particles between chip bumps and substrate pads can be approximated as the product of the total number of particles and the load estimated from Fig. 6 for specific deformation:

$$
F_{\text {total }}=N_{p}(\text { particles }) \times F_{p}(\text { load } / \text { particle })
$$

where $N_{p}$ is the total number of particles under compression and $F_{p}$ is the load required to compress a single conductive particle for the specific deformation displacement.

Fig. 9 shows the cross section images of conductive particles deformed between a bump and a substrate pad under various bonding forces. Table I summarizes the relationship between estimated particle deformation degree and the measured deformation degree under the various bonding forces. The actual particle deformation degree well agrees with the predicted values based on the load versus deformation results for specific contact

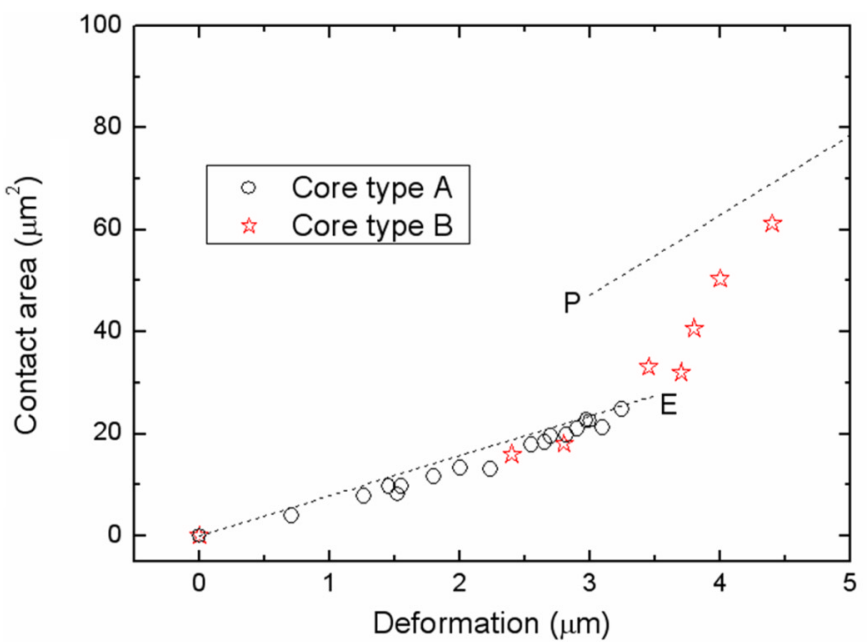

(a)

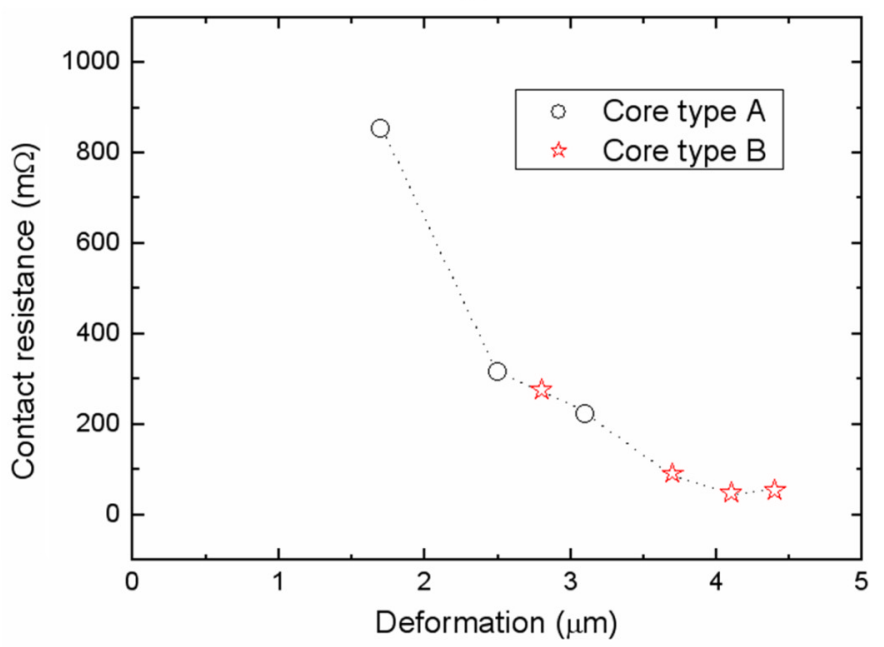

(b)

Fig. 10. Changes of (a) contact area and (b) contact resistance as a function of deformation displacement. The dotted lines represent the theoretical models for elastic (marked with $E$ ) and full plastic deformation (marked with $P$ ), respectively.

force. Even under the same bonding force, deformation degree of conductive particles was significantly dependent upon the polymer core material. The bonding force for an ACA package with arbitrary I/O number, bump area, and conductive particle density can be quantitatively estimated by using the equivalent spring model and load-deformation result for specific deformation degree.

\section{Mechanical and Electrical Contact Analysis of Conductive Particles}

In this part, both mechanical contact behaviors such as deformation degree and contact area and electrical contact resistance are investigated as functions of core material and bonding force. As described above, when a bonding load is applied on conductive particles between chip bumps and substrate pads, the particle becomes flattened and contact area increases. Moreover, under the same bonding load, two types of conductive particle obviously exhibited the different deformation degree. By examining the optical view and cross-sectional view of deformed particles, contact characteristics such as deformation degree and contact area were numerically obtained. Fig. 10 shows 


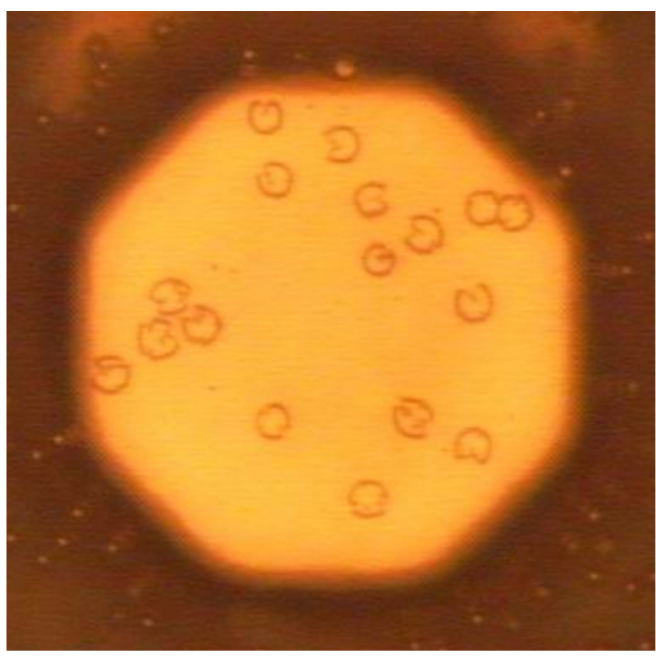

(a)

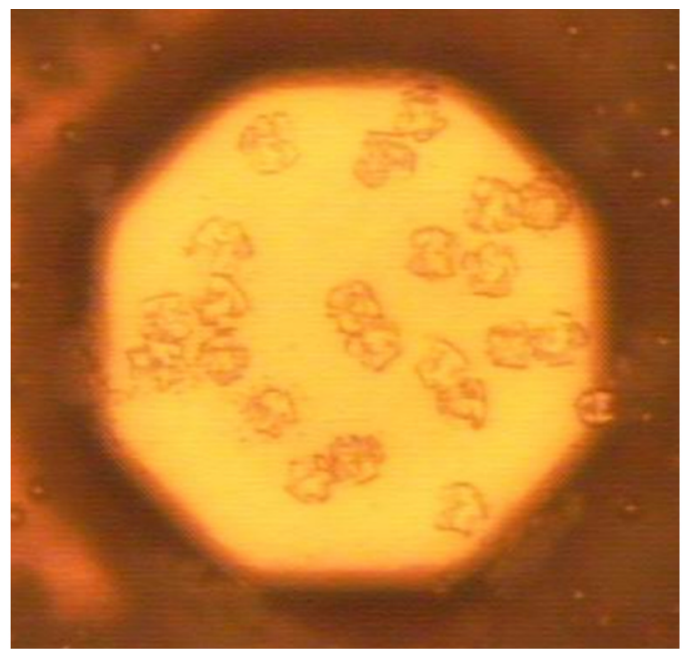

(b)

Fig. 11. Optical pictures of deformed conductive particles under different deformation degree. The cracking of the coating layer caused by significant particle deformation resulted in the contact resistance increase.

the contact area and contact resistance as a function of deformation degree. The dotted lines in Fig. 10(a) represent the theoretical models estimated by (5) and (6) for elastic deformation and fully plastic deformation, respectively. It is observed that in the region of particle deformation below $3.5 \mu \mathrm{m}$, the change of contact area closely follows the elastic deformation behavior for two types of core material. Regardless of different compression hardness for core material $\mathrm{A}$ and $\mathrm{B}$, the same deformation degree resulted in the same contact area. As the conductive particle deformed beyond $3.5 \mu \mathrm{m}$, contact area was deviated from the elastic deformation model and asymptotically approach the fully plastic deformation behavior in Fig. 10(a). It reveals that above deformation degree of $3.5 \mu \mathrm{m}$, plastic deformation fully becomes dominant within the conductive particle. Presumably, a sudden drop of contact resistance at about 3.6- $\mu \mathrm{m}$ deformation in Fig. 10(b) may be due to the abrupt increase of contact area. However, the significant increase of contact area above deformation degree of $4.0 \mu \mathrm{m}$ did not remarkably lower the contact resistance. It is because the continuous decrease of contact resistance with increasing load is counterbalanced by the breaking of metal coating layer of conductive particle as shown in Fig. 11. This rupture of the metal coating layer under the large particle deformation was also found in cross-sectional images of Fig. 9, indicated by the discontinuity of the coating layer. That is, the bonding force is too high and the particles have been crushed, resulting in the contact resistance increase.

Fig. 12 shows the contact area and contact resistance as a function of bonding force. As bonding force increased, contact resistance decreased due to the increase of deformation degree and contact area. Moreover, under the same bonding force, the contact resistance and its deviation of an ACF with B-type particle core were remarkably lower than those of an ACF with A-type core. It is because the soft conductive particle has larger deformation degree and contact area than hard one under the same bonding load.

One interesting observation is the minor increase of contact resistance in the vicinity of $150 \mathrm{~N}$. As described above, this is due to the separation or cracking of surface gold coating layer of

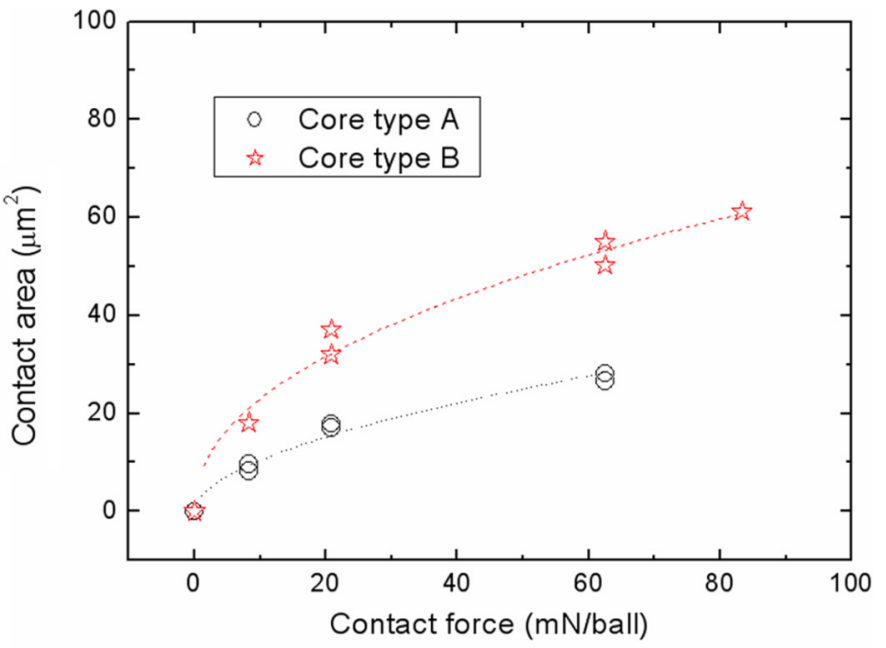

(a)

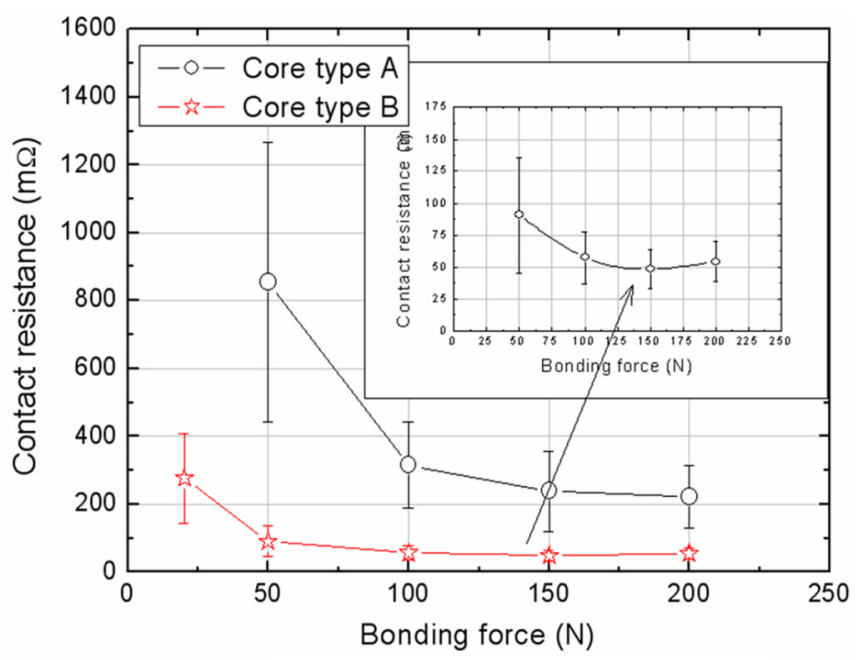

(b)

Fig. 12. Changes of (a) contact area and (b) contact resistance as a function of bonding force. 
conductive particles caused by significant particle deformation under the excessive contact load.

\section{CONCLUSION}

The load-deformation behavior of a single conductive particle for anisotropic conductive adhesive interconnection was quantitatively measured by using a nano-mechanical measurement technique. It was also clearly shown that the deformation degree of a conductive particle was significantly dependent upon the polymeric core material of a conductive particle as well as the bonding force. The bumped silicon chips were mounted on glass substrates using ACA under various bonding force. By comparing the changes of deformation degree as functions of bonding force and core material obtained experimentally and computationally, it was concluded that the measured load-deformation curve could provide a sophisticated approach for ACA interconnection. That is, the bonding force for ACA interconnection with an arbitrary I/O number, bump area, and conductive particle density can be quantitatively predicted by using the equivalent spring model and load-deformation result. In the vicinity of $70 \%$ deformation degree $(5-\mu \mathrm{m}$ particle diameter), the contact area between a deformed particle and a bump increased rapidly due to the plastic deformation within the polymer core, which resulted in the abrupt decrease of contact resistance. However, the excessive particle deformation caused the bounce up in the contact resistance. It has thus been concluded that the load-deformation behavior of a single conductive particle and its influence on the electrical conduction becomes a critical factor in the design and optimization of the reliable ACA interconnection.

\section{REFERENCES}

[1] J. Liu, "An overview of advances of conductive adhesive joining technology in electronics applications," Mater. Technol., vol. 10, pp. 247-252, 1995.

[2] M. J. Yim et al., "A study on the electrical conduction mechanism of anisotropically conductive film for LCD packaging application," in $\mathrm{Ad}$ vances in Electronic Packaging. New York: EEP, 1997, vol. 19-1, pp. $65-72$.

[3] Y. Fu et al., "Experimental and theoretical characterization of electric contact in anisotropically conductive adhesive," IEEE Trans. Comp., Packag., Manufact. Technol. B, vol. 23, no. 1, pp. 15-21, Feb. 2000.

[4] Z. Lai et al., "Anisotropically conductive adhesive flip-chip bonding on rigid and flexible printed circuit substrate," IEEE Trans. Comp., Packag., Manufact. Technol. B, vol. 19, no. 3, pp. 644-660, Aug. 1996.
[5] W. S. Kwon et al., "Fundamental understanding of ACF conduction establishment with emphasis on the thermal and mechanical analysis," Int. J. Adhes. Adhes., vol. 24, pp. 135-142, 2004.

[6] W. S. Kwon et al., "Contraction stress build-up of anisotropic conductive films (ACF's) for flip-chip interconnection: Effect of thermal and mechanical properties of ACFs," J. Appl. Polym. Sci., vol. 93, pp. 2634-2641, 2004.

[7] K. L. Johnson et al., Contact Mechanics. Cambridge, U.K.: Cambridge Univ. Press, 1987.

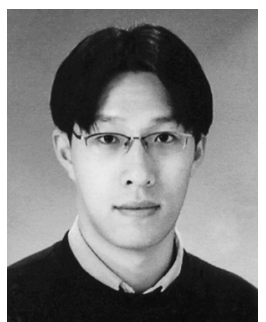

Woon-Seong Kwon received the B.S. and M.S. degrees in material science and engineering from the Korea Advanced Institute of Science and Technology (KAIST), Daejon, in 1998 and 2001, respectively, where he is currently pursuing the Ph.D. degree in microelectronic packaging.

His research interests are the development of anisotropic conductive adhesive materials for packaging applications, advanced flip-chip packages using polymeric materials, and its thermomechanical reliability assessment and high frequency electrical characterization.

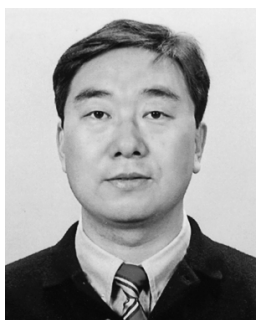

Kyung-Wook Paik (M'90) received the B.S. degree in metallurgical engineering from Seoul National University, Seoul, Korea, in 1979, the M.S. degree from the Korea Advanced Institute of Science and Technology (KAIST), Taejon, in 1981, and the Ph.D. degree in materials science and engineering from Cornell University, Ithaca, NY, in 1989.

From 1982 to 1985 , he was with KAIST, Seoul, as a Research Scientist and was responsible for various materials development such as gold bonding wire and nonferrous alloys. After the Ph.D. degree, he worked at General Electric Corporate Research and Development from 1989 to 1995 , where he was involved with the R\&D of materials and processes of GE high density interconnect (HDI) multichip module technology and power I/C packaging as a Senior Technical Staff. He next joined KAIST in 1995, and has been working in the Department of Materials Science and Engineering as an Associate Professor. He is currently working in the area of MCM, flip chip, MEMS, and display packaging. He had visited the Packaging Research Center, Georgia Institute of Technology, Atlanta, as a Visiting Professor from March 1999 to February 2000, and was involved in the educational and integrated passives research programs. He has published more than 50 technical papers in the area of electronic packaging and currently holds ten U.S. patents and has four U.S. patents pending.

Dr. Paik is the Chairman of the Korean IEEE-CPMT Chapter and also a member of the Materials Research Society (MRS) and IMAPS. 